\title{
Multi-level Pseudo-Random Signal Design and "Model-on-Demand" Estimation Applied to Nonlinear Identification of a RTP Wafer Reactor
}

\author{
M.W. Braun, D.E. Rivera, A. Stenman', W. Foslien, and C. Hrenya \\ ${ }^{\dagger}$ Department of Electrical Engineering \\ Linköping University, SE-581 83 Linköping, Sweden \\ URL: http://www . control . isy. liu.se \\ Email: stenman@isy.liu.se
}

June 15, 1999

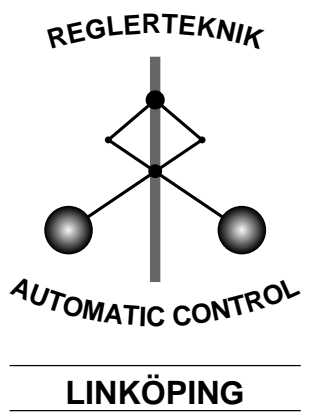

Report no.: LiTH-ISY-R-2156

Accepted for presentation at ACC '99, San Diego

Technical reports from the Automatic Control group in Linköping are available by anonymous ftp at the address ftp.control.isy.liu.se. This report is contained in the PDF file 2156.pdf. 


\title{
Multi-level Pseudo-Random Signal Design and "Model-on-Demand" Estimation Applied to Nonlinear Identification of a RTP Wafer Reactor
}

\author{
M. W. Braun*, D. E. Rivera* ${ }^{1}$, A. Stenman**, W. Foslien***, and C. Hrenya*** \\ *Department of Chemical, Bio, and Materials Engineering \\ Control Systems Engineering Laboratory, Manufacturing Institute \\ Arizona State University, Tempe, Arizona 85287-6006 \\ ** Division of Automatic Control, Department of Electrical Engineering \\ Linköping University, 5-581 83 Linköping, Sweden \\ *** Honeywell Technology Center \\ 3660 Technology Drive, Minneapolis, MN 55418
}

\begin{abstract}
Guidelines are presented for specifying the design parameters of multi-level pseudo-random sequences in a manner useful for "plant-friendly" nonlinear system identification. These multi-level signals are introduced into a Rapid Thermal Processing wafer reactor simulation and compared against a well-designed pseudorandom binary sequence (PRBS). The resulting data serves as a database for a "Model on Demand" (MoD) predictor. MoD estimation is attractive because it requires less engineering effort to model a nonlinear plant, compared to global nonlinear models such as neural networks. The improved fit of multi-level signals over the PRBS signal, as well as the usefulness of the MoD estimator, is demonstrated on validation data.
\end{abstract}

\section{Introduction}

Pseudo-random binary sequences (PRBS) are widely used in the identification of linear systems. The advantages of the PRBS input include ease of implementation and an autocorrelation function similar to white noise. Since the PRBS is periodic and deterministic, it can be designed to possess excitation in a control-relevant frequency range over a single data cycle. Because the PRBS can be applied to a process multiple times, it provides the user with a convenient means for discarding corrupted segments of data and retaining the most informative data for model estimation and validation.

\footnotetext{
${ }^{1}$ To whom all correspondence should be addressed. phone: (602) 965-9476; e-mail: daniel.rivera@asu.edu
}

The PRBS input, however, is not always well suited for nonlinear problems. Since the PRBS consists of only two levels, the resulting data may not provide sufficient information to identify nonlinear behavior (e.g. $\left.y(k)=u^{2}(k)\right)$. Additionally, a PRBS signal of too large a magnitude may bias the estimation of the linear kernel. Multi-level pseudo-random sequences (m-level PRS), in contrast, allow the user to highlight nonlinear system behavior while manipulating the harmonic content of the signal to enable unbiased estimation of the linear dynamics in the presence of nonlinearities [5]. In this paper the theoretical framework developed by Barker and Zhuang [1] is extended to include the use of a priori knowledge of the physical system and the goals of the control problem. "Plant-friendly" design of these multi-level PRS inputs is accomplished by incorporating a priori knowledge available to the engineer (such as the estimated dominant time constant and closedloop control requirements) into values for the design parameters of the input signal. The results are philosophically similar to the work performed by Gaikwad and Rivera [4] for PRBS and Schroeder-phased multisinusoidal input design.

Various realizations of m-level PRS signals designed according to these guidelines are used to generate a database for a "Model on Demand" (MoD) predictor. "Model on Demand" or "Just-In-Time" (JIT) estimation is a novel paradigm first proposed by Cybenko [3]. The philosophy of MoD modeling is that a model is not estimated until it is really needed. All observations of the process are stored in a database, which is accessed to estimate a local model at the current operating point. The variance/bias tradeoff is optimized locally by adapting the number of data and their relative 
weighting. The MoD approach enhances local modeling and provides a reasonable alternative to nonlinear black-box techniques that optimize model predictions globally (such as neural networks). The MoD modeling formulation pursued in this paper follows from the approach of Stenman and co-workers $([7,8])$. The predictive ability of the MoD estimator is demonstrated on a Rapid Thermal Processing (RTP) reactor model developed by Honeywell Technology Center. The MoD results are evaluated for both m-level PRS inputs and a well designed PRBS signal.

\section{Generation of m-level PRS Signals}

The theory behind the generation and application of m-level PRS inputs in system identification is well developed [5]. m-level PRS signals are generated in a similar manner to the PRBS using shift registers and modulo addition. As with the PRBS, m-level PRS inputs are periodic, deterministic signals and have an autocorrelation function similar to white noise.

Recent work by Barker and coworkers develops theoretical methods for generation and design of m-level PRS signals with suppressed harmonics of multiples 2, 3 and 5. [1]. The basis for generation of these m-level PRS signals lies in finite field theory. A Galois field (GF), defined by $q$ elements, guides the creation of a pseudo-random sequence, $\left(x_{1} \ldots x_{i}\right)$, from a shift register. Note that $q$ may be a prime number or the power of a prime number $\left(q=p^{k}\right)$. The coefficients $c_{i}$ for the outputs of the stages of the shift register are derived from a primitive polynomial $f(x)$ in $G F(q)$. Figure 1 shows the shift register configuration for m-level PRS generation. Once the sequence is generated it is then

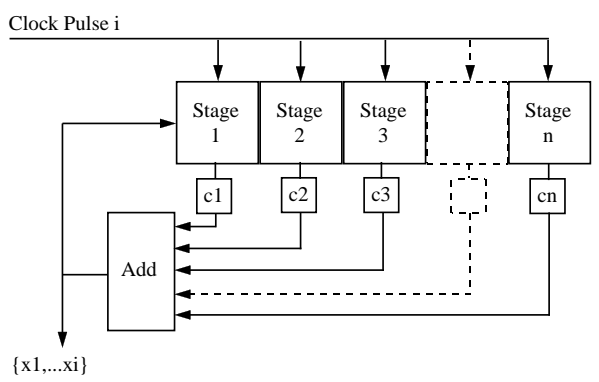

Figure 1: m-level PRS generation algorithm

scaled or "mapped" to the relative levels of the input, based on the Galois field chosen, the multiple of harmonics to be suppressed, and the number of terms in the candidate model [1]. Peak factor and uniformity of the power spectrum are also considerations when choosing mappings. Once the signal is mapped, it is then scaled with an acceptable amplitude and each element in the sequence is introduced into the plant after a predetermined switching time $T_{s w}$ has passed.

\section{3 m-level PRS Design Guidelines}

The user of m-level PRS signals is faced with the task of judiciously selecting the design parameters needed to generate the signal. This section describes how to use the following prior information

- Desired closed-loop speed of response.

- Estimated range of dominant time constant.

- Order of nonlinearity of plant and order of model to be fit.

- Acceptable signal length and amplitude.

to systematically arrive at values for the design variables. Figure 2 summarizes the design procedure. In some cases, the user may be required to ease constraints on signal amplitude or signal length in order to meet identification objectives.

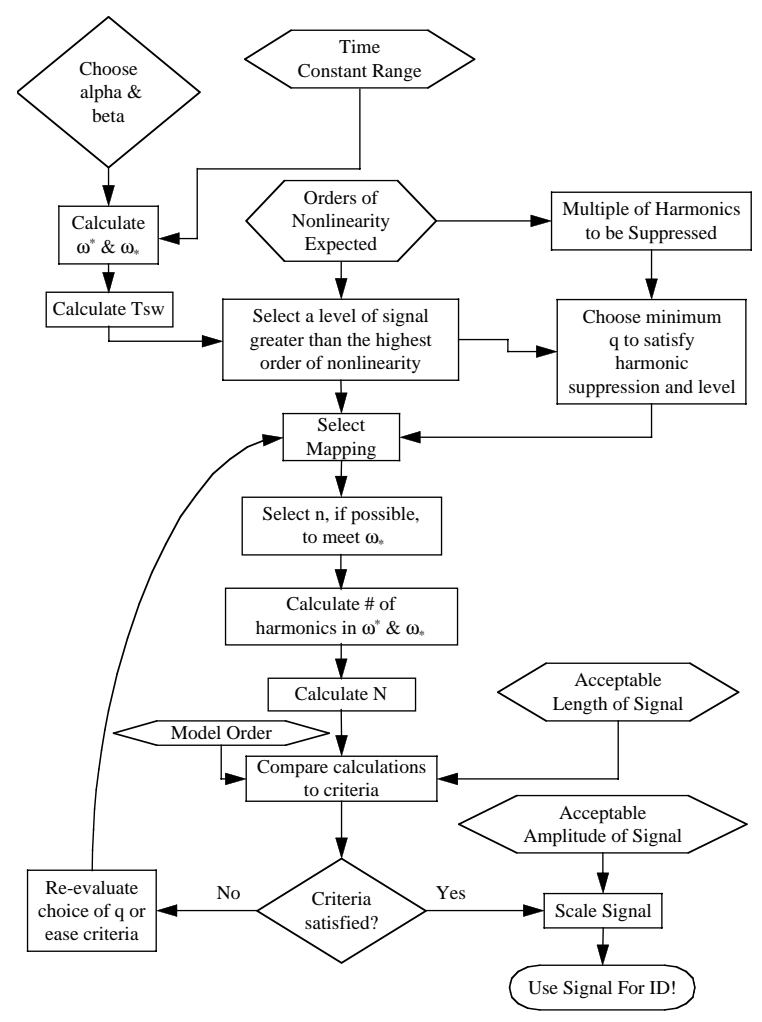

Figure 2: Flowchart for multi-level PRS signal design

A priori knowledge of the dominant time constant range $\left(\tau_{d o m}^{H}\right.$ to $\left.\tau_{d o m}^{L}\right)$ makes it possible to place the 
power of the input signal in the frequency range important for control. The frequency range of interest (corresponding to that between the first harmonic and the bandwidth of the power spectrum) should fall within the range specified in Equation 1:

$$
\omega_{*}=\frac{1}{\beta \tau_{d o m}^{H}} \leq \omega \leq \frac{\alpha}{\tau_{d o m}^{L}}=\omega^{*}
$$

The parameters $\alpha$ and $\beta$ define the desired range for high and low frequency excitation. $\beta$ (normally equal to 3,4 , or 5 ) specifies low frequency information corresponding to the $95 \%$ settling time, $98 \%$ settling time, and $99 \%$ settling time, respectively of the open-loop plant. $\alpha$ determines the high frequency content in the signal and is defined on the basis of how much faster the intended closed-loop speed of response will be relative to open-loop. Typical values chosen for $\alpha$ and $\beta$ are 2, and 3, respectively. However, as the plant of interest or operating region becomes more nonlinear, the frequency range of the input signal is not as important as the shape of the input signal, as noted by Pearson and Ogunnaike [6]. $\alpha$ and the number of levels may be used to adjust the shape of the signal.

The number of levels $m$ should be at least one greater than the nonlinearity order in the candidate model. The length of the Galois field $q$ must support the required suppression of harmonics. Harmonics can be suppressed in multiples of $2(q \geq 3), 2$ and $3(q \geq 7)$, and 2,3 , and $5(q \geq 31)$. $q$ must always be greater or equal to the required number of levels $(q \geq m)$. Estimates of the switching time, $T_{s w}$, and number of shift registers, $n_{r}$, can be made from Equation 2 .

$$
T_{s w} \leq \frac{2.78}{\omega^{*}}, \quad \omega_{*} \geq \frac{2 \pi}{T_{s w}\left(q^{n_{r}}-1\right)}
$$

The number of nonsuppressed harmonics present within the frequency range of interest can be calculated via Equation 3:

$$
\# \text { of harm. }=\frac{2.78}{2 \pi}\left(q^{n_{r}}-1\right) \gamma
$$

$\gamma$ is defined on the basis of the suppressed harmonics. For harmonics of multiple of 2 suppressed, $\gamma=1 / 2$. For harmonics of multiples of 2 and 3 suppressed, $\gamma=1 / 3$. For harmonics of multiples of 2,3 and 5 suppressed, $\gamma=4 / 15$. Note that for simultaneously low values of $n_{r}$ (e.g. 2) and $q$ (e.g. 3 ), this equation provides only a rough estimate. To increase the number of nonsuppressed frequencies in the signal (to allow for persistent excitation of the linear dynamics of the candidate model), the user can increase the values of $\alpha$ and $\beta$ in Equation 1 or equivalently decrease switching time or increase the number of shift registers.

Equation 4 determines the number of elements in one cycle of the input signal

$$
N_{c y c}=\left(q^{n_{r}}-1\right)
$$

$T_{c y c}=N_{c y c} * T_{s w}$ specifies the signal duration for one cycle. If signal duration represents a significant constraint in the input design problem, then the multiples of harmonics to be suppressed and the frequency range of the signal may have to be compromised in order to reduce the length of the signal.

Finally, it is important to choose a "plant-friendly" amplitude for the signal. Since an m-level PRS signals can switch from minimum to maximum values in one switching time, the acceptable amplitude span must be selected to avoid actuator restrictions, product quality constraints, move size restrictions, and so forth.

\section{Model-on-Demand Prediction}

Consider a SISO process with nonlinear ARX structure, i.e.,

$$
y(k)=m(\varphi(k))+e(k), k=1, \ldots, M
$$

where $m(\cdot)$ is an unknown nonlinear mapping and $e(k)$ is an error term modeled as i.i.d. random variables with zero mean and variance $\sigma_{k}^{2}$. The MoD predictor attempts to estimate a value $\hat{y}$ based on a local neighborhood of the regressor space $\varphi(t)$ (Stenman [7]). The regressor vector is of the form

$$
\begin{aligned}
\varphi(t)= & {\left[y(t-1) \ldots y\left(t-n_{a}\right)\right.} \\
& \left.u\left(t-n_{k}\right) \ldots u\left(t-n_{b}-n_{k}\right)\right]^{T}
\end{aligned}
$$

where $n_{a}, n_{b}$, and $n_{k}$ denote the number of previous outputs, inputs, and delays in the model.

A local estimate $\hat{y}$ can be obtained from the solution of the weighted regression problem

$$
\begin{array}{r}
\hat{\beta}=\arg \min _{\beta} \sum_{k=1}^{N} \ell(y(k)-m(\varphi(k), \beta)) \\
\cdot W\left(\frac{\|\varphi(k)-\varphi(t)\|_{M}}{h}\right),
\end{array}
$$

where $\ell(\cdot)$ is a scalar-valued and positive norm function, $\|u\|_{M} \triangleq \sqrt{u^{T} M u}$ is a scaled distance function on the regressor space, and $h$ is a bandwidth parameter controlling the size of the local neighborhood, and $W(\cdot)$ is a window function (usually referred to as the kernel) assigning weights to each remote data point according to its distance from $\varphi(t)$. The window is typically a bell-shaped function with bounded support.

In principle, it is possible to use any nonlinear model structure as a local model in Equation 7. However, if a quadratic norm, $\ell(\varepsilon)=\varepsilon^{2}$ is used and the model is linear in the unknown parameters, the estimate can be easily computed using simple and powerful least 
squares methods. We thus assume a local linear model structure,

$$
m(\varphi(k), \beta)=\beta_{0}+\beta_{1}^{T}(\varphi(k)-\varphi(t))
$$

as the default choice in the rest of the paper. If $\hat{\beta}_{0}$ and $\hat{\beta}_{1}$ denote the minimizers of (7) using the model from (8), a one-step ahead prediction of $y(t)$ is given by

$$
\hat{y}(t)=m(\varphi(t), \hat{\beta})=\hat{\beta_{0}} .
$$

Each local regression problem produces a single prediction $\hat{y}(t)$ corresponding to the current regression vector $\varphi(t)$. To obtain predictions at other locations in the regressor space, the weights change and new optimization problems have to be solved. This is in contrast to the global modeling approach where the model is fitted to data only once. However, in a neighborhood around $\varphi(t)$, the local linear model per Equation 8 provides an input-output linearization of the form

$$
A\left(z^{-1}\right) y(k)=B\left(z^{-1}\right) u\left(k-n_{k}\right)+\alpha .
$$

where $A\left(z^{-1}\right)$ and $B\left(z^{-1}\right)$ are polynomials in the backward time-shift operator $z^{-1}$ obtained from the components of $\hat{\beta}_{1}$, and $\alpha=\hat{\beta}_{0}-\hat{\beta}_{1} \varphi(t)$ is an offset term.

The bandwidth $h$ controls the neighborhood size and has a critical impact on the resulting estimate since it governs a trade-off between the bias and variance errors of the estimate. Traditional bandwidth selectors produce a single global bandwidth; in MoD estimation, bandwidth is computed adaptively at each prediction. While various measures are available for this purpose [8], the method used in this paper is the Akaike's Final Prediction Error (FPE).

\section{Case Study}

Honeywell Technology Center has developed a simplified model of a RTP wafer reactor [2]. Figure 3 shows a sketch of the configuration. The five inputs to the system are the percent power to each lamp, ranging from 0 to $100 \%$. The five outputs of the system are the temperatures $[\mathrm{K}]$ of the wafer directly above the lamps. A simplified model examined by Honeywell is shown in Equation 11. This model displays an RMS error of $1.2 \mathrm{~K}$ in comparison to the true plant. Gaussian noise with a variance of $4.2 \%$ and a sampling time of 0.2 seconds was added to the percent power input signal of our true plant. Our approach is to use the simulation model of Equation 11, with the parameters provided by Honeywell, as our plant model. We are focused on SISO identification of lamp \#2.

$$
\begin{aligned}
y_{k}= & P_{1} \cdot y_{k-1}+P_{2} \cdot\left(\frac{y_{k-1}}{1000}\right)^{4}+P_{3} \cdot\left(\frac{y_{k-1}}{1000}\right)^{3} \\
& +P_{4} \cdot\left(\frac{y_{k-1}}{1000}\right)^{2}+P_{5} \cdot\left(\frac{u_{k-1}}{100}\right)^{4}+P_{6} \cdot\left(\frac{u_{k-1}}{100}\right)^{3} \\
& +P_{7} \cdot\left(\frac{u_{k-1}}{100}\right)^{2}+P_{8} \cdot u_{k-1}+P_{9}
\end{aligned}
$$
date m-level PRS signals are summarized in Table 1. The regressor space for the PRBS data could not be adjusted for a reasonable fit. The resulting fits for the best and worst-fit case m-level PRS signals considered

The design of multi-level PRS signals begins by estimating the range of $\tau_{d o m}$. Through a series of short negative and positive step tests distributed throughout the input signal range, $\tau_{d o m}^{H}$ was estimated to be $33 \mathrm{sec}-$ onds and $\tau_{d o m}^{L}$ was estimated to be 7 seconds. $\alpha=2$ and $\beta=6$ were chosen to insure the low frequency content of the signal. The switching time for both the PRBS and the multi-level PRS signals was calculated to be less than 9.73 seconds. A $T_{s w}$ of 9 seconds was selected which meets the high frequency bound. For the PRBS, 7 shift registers are needed to meet the low frequency requirements, translating into a PRBS signal of 1143 seconds, per the guidelines in Gaikwad and Rivera [4].

For the multi-level signals, a variety of combinations are possible which meet the guidelines; some of these are summarized in Table 1. In all cases presented $q=13$ and $n_{r}=2$ which satisfied the low frequency limit while keeping signal length at a minimum (1512 seconds). Figures 4 and 5 show the time and frequency domain representations, respectively, for a 13-level signal with harmonics of multiple 2 suppressed. We evaluated 2 other variations of the signal, one with harmonics of 2 and 3 suppressed and one using only 5 levels. We chose to excite the plant between $5 \%$ and $99 \%$ of the lamp power, allowing an approximate output range between $730 K$ and $1425 K$. The validation data (Figure 6) was independently designed, based on temperature ramp rates and processing times normally experienced with this reactor [2]. The validation data exhibits ramp rates from $27.8 \mathrm{~K} / \mathrm{sec}$ to $71.7 \mathrm{~K} / \mathrm{sec}$ and processing times from $20 \mathrm{sec}$ to $120 \mathrm{sec}$.

The estimation was performed on only one period of each of the signals and simulation performed on the validation data set. The regressor space $\left[n_{a} n_{b} n_{k}\right]$ which provided the lowest RMS fit was used for comparison of the signals. The fits for the PRBS and the candi- 
a.)
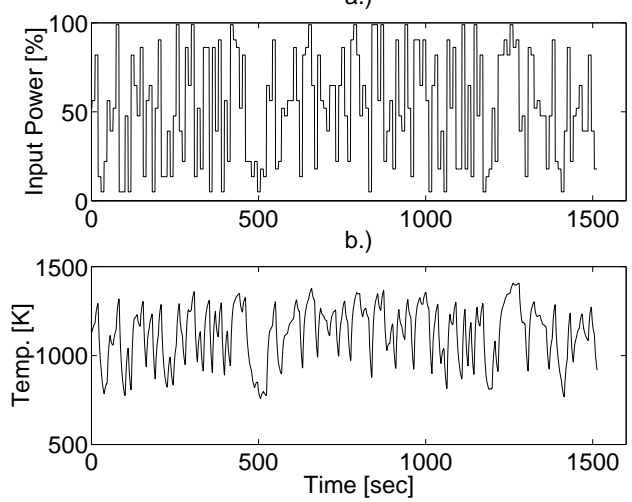

Figure 4: a.) 13 level PRS input ( $\left.\mathrm{q}=13, \mathrm{~h}=2 \& n_{r}=2\right)$ b.) corresponding output from RTP reactor

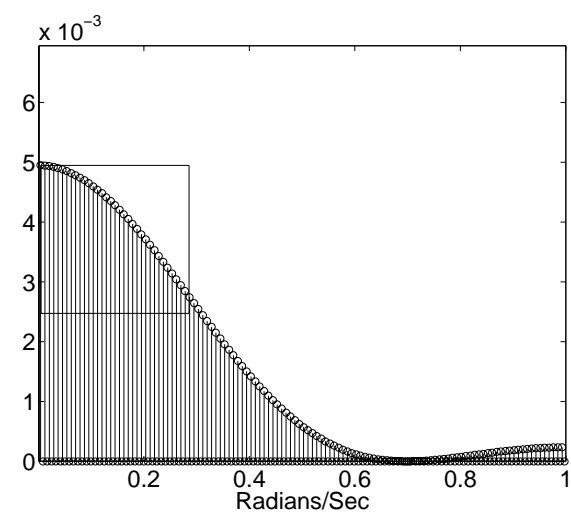

Figure 5: Power spectrum for 13 level PRS in Figure 4 with control-relevant region emphasized

are presented in Figure 6.

Table 1: RMS Fits on RTP Validation Data

\begin{tabular}{ccccc}
$\begin{array}{c}\text { Galois } \\
\text { Field } q:\end{array}$ & $\begin{array}{c}\text { Level: } \\
m\end{array}$ & $\begin{array}{c}\text { Harmonics } \\
\text { Sup'd (h): }\end{array}$ & $\begin{array}{c}\text { RMS } \\
\text { Fit }(\mathrm{K}):\end{array}$ & $\begin{array}{c}\text { ARX } \\
n_{a} n_{b} n_{k}:\end{array}$ \\
\hline 2 (PRBS) & 2 & - & 53909.1 & 1241 \\
13 & 13 & 2 & 16.6 & 171 \\
13 & 13 & $2 \& 3$ & 16.7 & 1171 \\
13 & 5 & 2 & 54.0 & 1341
\end{tabular}

\section{Conclusions}

Guidelines for the systematic design of multi-level PRS signals have been developed for control-relevant, plantfriendly identification of nonlinear plants. The guidelines utilize a priori knowledge to specify the design parameters for this signal. The resulting database is rich enough to support a MoD based predictor; its effectiveness is illustrated in an RTP reactor simulation example.

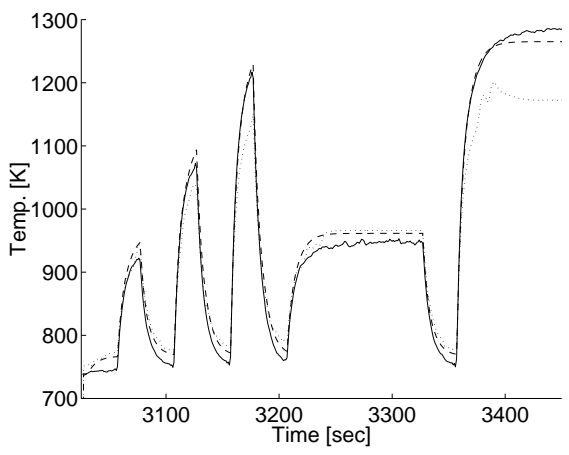

Figure 6: Solid:Validation Data, Dashed: MoD for $m=13$, $\mathrm{h}=2$; Dotted: MoD for $m=5, \mathrm{~h}=2$

Acknowledgment. We would like to thank Prof. H. A. Barker of University College of Swansea, UK for the use of his Galois software.

\section{References}

[1] H. A. Barker and M. Zhuang. "Design of pseudorandom perturbation signals for frequency-domain identification of nonlinear systems." 11th IFAC Symp. on System Ident., 3, 1997.

[2] J. Christoffel, W. Foslien, A. Mathur, and M. Ekblad. "Model Based Control and Optimization Techniques from the Advanced Materials Industry Applied to Semiconductor Processes", AEC/APC Workshop VII, pgs. 157-172, November 1995.

[3] G. Cybenko, Just-in-time learning and estimation. In S. Bittani and G. Picci, editors, Identification, Adaptation, Learning, NATO ASI series, pages 423-434, Springer, 1996.

[4] S. V. Gaikwad and D. E. Rivera. Control-relevant input signal design for multivariable system identification: Application to high-purity distillation. In: Proceedings of the 13th IFAC World Congress:IFAC'96. Vol. M. pp. 349-354, 1996.

[5] K. Godfrey. Perturbation Signals For System Identification. Prentice Hall International (UK) Limited, Hertfordshire UK, 1993.

[6] R. K. Pearson and B. A. Ogunnaike. Nonlinear Process Control. M. A. Henson and D. E. Seborg Editors, Prentice Hall PTR, Upper Saddle River, New Jersey. 1997.

[7] A. Stenman, F. Gustafsson, and L. Ljung. "Just in time models for dynamical systems." In Proceedings of the 35th IEEE Conference on Decision and Control, Kobe, Japan, 1996.

[8] A. Stenman. "Just-in-Time Models with Applications to Dynamical Systems." Licentiate thesis LIUTEK-LIC-1997:02. Department of Electrical Engineering, Linköping University. S-581 83 Linköping, Sweden. 